\title{
A G protein-coupled, IP3/protein kinase C pathway controlling the synthesis of phosphaturic hormone FGF23
}

Qing He, ${ }^{1}$ Lauren T. Shumate, ${ }^{1}$ Julia Matthias, ${ }^{1}$ Cumhur Aydin, ${ }^{1,2}$ Marc N. Wein, ${ }^{1}$ Jordan M. Spatz, ${ }^{1}$ Regina Goetz, ${ }^{3}$ Moosa Mohammadi, ${ }^{3}$ Antonius Plagge, ${ }^{4}$ Paola Divieti Pajevic, ${ }^{5}$ and Murat Bastepe ${ }^{1}$

${ }^{1}$ Endocrine Unit, Department of Medicine, Massachusetts Ceneral Hospital and Harvard Medical School, Boston, Massachusetts, USA. ${ }^{2}$ Department of Endodontics, Gulhane Faculty of Dentistry, University of Health Sciences, Ankara, Turkey. ${ }^{3}$ Department of Biochemistry \& Molecular Pharmacology, New York University School of Medicine, New York, New York, USA. ${ }^{4}$ Department of Cellular and Molecular Physiology, Institute of Translational Medicine, University of Liverpool, Liverpool, United Kingdom. ${ }^{5}$ Department of Molecular \& Cell Biology, Boston University Goldman School of Dental Medicine, Boston, Massachusetts, USA.

Dysregulated actions of bone-derived phosphaturic hormone fibroblast growth factor 23 (FGF23) result in several inherited diseases, such as X-linked hypophosphatemia (XLH), and contribute substantially to the mortality in kidney failure. Mechanisms governing FGF23 production are poorly defined. We herein found that ablation of the $G_{q / 11} \alpha$-like, extralarge $G \alpha$ subunit (XL $\left.\alpha s\right)$, a product of CNAS, exhibits FGF23 deficiency and hyperphosphatemia in early postnatal mice (XLKO). FGF23 elevation in response to parathyroid hormone, a stimulator of FGF23 production via CAMP, was intact in XLKO mice, while skeletal levels of protein kinase C isoforms $\alpha$ and $\delta$ (PKC $\alpha$ and PKC $\delta$ ) were diminished. XLas ablation in osteocyte-like Ocy454 cells suppressed the levels of FGF23 mRNA, inositol 1,4,5-trisphosphate (IP3), and PKC $\alpha /$ PKC $\delta$ proteins. PKC activation in vivo via injecting phorbol myristate acetate (PMA) or by constitutively active Gq $\alpha$-Q209L in osteocytes and osteoblasts promoted FGF23 production. Molecular studies showed that the PKC activationinduced FGF23 elevation was dependent on MAPK signaling. The baseline PKC activity was elevated in bones of Hyp mice, a model of XLH. XLas ablation significantly, but modestly, reduced serum FGF23 and elevated serum phosphate in Hyp mice. These findings reveal a potentially hithertounknown mechanism of FCF23 synthesis involving a G protein-coupled IP3/PKC pathway, which may be targeted to fine-tune FGF23 levels.

Conflict of interest: MNW's laboratory receives research support from Radius Health and Galapagos NV for projects unrelated to this manuscript.

Copyright: (c) 2019, American Society for Clinical Investigation.

Submitted: September 20, 2018 Accepted: August 1, 2019 Published: September 5, 2019

Reference information: /CI Insight. 2019:4(17):e125007. https://doi.org/10.1172/jci. insight.125007.

\section{Introduction}

Phosphate is necessary for cell metabolism and growth, as well as skeletal development and mineralization, and excess levels of phosphate can lead to soft tissue mineralization (1-5). Fibroblast growth factor 23 (FGF23) is a polypeptide hormone that acts on kidneys to stimulate urinary phosphate excretion and to inhibit the generation of the bioactive vitamin $\mathrm{D}$ metabolite, 1,25 dihydroxyvitamin $\mathrm{D}\left[1,25(\mathrm{OH})_{2} \mathrm{D}\right](6,7)$. FGF23 deficiency or impaired FGF23 action results in increased levels of serum phosphate, $1,25(\mathrm{OH})_{2} \mathrm{D}$, and calcium, thus causing increased tendency of soft tissue mineralization, as seen in certain forms of tumoral calcinosis. Conversely, excess production of FGF23 leads to renal phosphate wasting and hypophosphatemia with inappropriately suppressed levels of $1,25(\mathrm{OH})_{2} \mathrm{D}$, as seen in tumor-induced osteomalacia and several inherited disorders of hypophosphatemia, such as X-linked hypophosphatemic rickets. FGF23 production also increases markedly in iron deficiency, driving the hypophosphatemia in patients with autosomal dominant hypophosphatemic rickets. In addition, the progressive increase of FGF23 in renal failure disrupts vitamin D metabolism, contributes to secondary hyperparathyroidism, and leads to impaired bone mineralization. Moreover, FGF23 elevation is directly associated with cardiovascular mortality seen in patients with chronic kidney disease. Recently, high circulating levels of FGF23 have also been associated with increased risk of dementia and Alzheimer's disease (8).

FGF23 is mainly synthesized by mature osteoblasts and osteocytes in bone $(9,10)$, but the molecular mechanisms and signaling pathways governing FGF23 production remain poorly understood. Several stimulators of 
FGF23 production have been identified, including dietary phosphorous, $1,25(\mathrm{OH})_{2} \mathrm{D}$, inflammatory cytokines, calcium, and iron deficiency $(1-5,11)$. In addition, skeletal production of FGF23 is also induced by FGF receptor 1 (FGFR1) signaling that primarily involves activation of the MAPK pathway (12-14). Another stimulator of FGF23 production is parathyroid hormone $(\mathrm{PTH})(15-18)$. The receptor for PTH (PTH1R) is a G proteincoupled receptor (GPCR), which couples to multiple heterotrimeric G proteins. The direct stimulatory action of PTH on FGF23 production, however, is mediated by the $\alpha$ subunit of the stimulatory G protein (Gs $\alpha$ ), thus involving cAMP generation.

Gs $\alpha$ is derived from the GNAS complex gene, which also gives rise to the extralarge Ga subunit (XL $\alpha$ ) (19). XLas uses a distinct, paternally active promoter and is partly identical to Gs $\alpha$, diverging from the latter in its N-terminal region $(20,21)$. When overexpressed in cells or in mice, XL $\alpha$ s mimics Gs $\alpha$ with respect to cAMP generation both basally and in response to activation of GPCRs, including PTH1R (22-24). Interestingly, XLas, despite sharing marked identity with $\mathrm{Gs} \alpha$, can mimic or enhance signaling of $\mathrm{G}_{\mathrm{q} / 11} \alpha$, another class of heterotrimeric $\mathrm{G} \alpha$ subunits, which stimulate phospholipase $\mathrm{C}$ and, thereby, trigger generation of inositol 1,4,5-trisphosphate (IP3) and diacylglycerol and the activation of protein kinase C (PKC) (25). $\mathrm{XL} \alpha \mathrm{s}$ is expressed in a wide range of tissues, including cells of the osteoblast lineage (26-28). In this study, we addressed whether FGF23 production is regulated by the cellular action of XLas. We found that XLas mediates FGF23 production in a cell-autonomous manner via the activation of PKC signaling. Our additional investigations using different mouse models and cell culture experiments showed that PKC activation is critically involved in FGF23 production, integrating signals from different intracellular pathways. We also revealed elevated PKC activity in the skeletal tissue of Hyp mice, a model of X-linked hypophosphatemia (XLH), and partially rescued the FGF23 and phosphate phenotype of those mice by ablating XLas.

\section{Results}

Serum FGF23 levels are significantly reduced in P10 XLas-knockout mice, with resultant increase in serum phosphate and 1,25(OH) $D$ levels. Serum phosphate levels have been found to be elevated in some pediatric patients with paternal GNAS defects and in 10-day-old XLas-knockout (XLKO) pups (29-36). We found that, at this age, XLKO pups were indeed hyperphosphatemic and, additionally, displayed modest hypocalcemia with only slightly elevated PTH levels that were not significantly different from the levels in WT littermates (Figure 1, A-C). In addition, both C-terminal and intact FGF23 levels were significantly reduced in XLKO mice at postnatal day 10 (P10) (Figure 1, D and E). Similarly, XLKO mice also had modestly reduced C-terminal FGF23 levels at 4 weeks and 2 months of age, compared with WT littermates, although the difference did not reach statistical significance (Supplemental Figure 1, A and B; supplemental material available online with this article; https://doi.org/10.1172/jci.insight.125007DS1). Moreover, serum level of 1,25(OH) $)_{2}$, whose synthesis is known to be suppressed by FGF23, was markedly increased in XLKO mice (Figure 1F). Quantitative reverse transcription PCR (qRT-PCR) analysis on total RNA isolated from P10 WT and XLKO femurs revealed that FGF23 mRNA levels in XLKO bone were less than $40 \%$ of the levels in WT bone (Figure $1 \mathrm{G}$ ). The expression levels of Phex and Dmp1, 2 genes in which inactivating mutations cause FGF23 excess and hypophosphatemic rickets, were also diminished in XLKO bones (Supplemental Figure 1C). 1,25(OH) ${ }_{2} \mathrm{D}$ level is controlled mainly by activities of the renal enzymes 25-hydroxyvitamin D 1 $\alpha$-hydroxylase (Cyp27b1), which synthesizes $1,25(\mathrm{OH})_{2} \mathrm{D}$, and 25-hydroxyvitamin D 24-hydroxylase (Cyp24a1), which metabolizes $1,25(\mathrm{OH})_{2} \mathrm{D}$. We found that the abundance of Cyp27b1 mRNA was increased over 2.7-fold, while the expression of Cyp24a1 mRNA was increased 1.8-fold in P10 XLKO kidneys compared with WT (Figure 1H), implying that the increased $1,25(\mathrm{OH})_{2} \mathrm{D}$ levels may reflect enhanced synthesis. FGF23 acts to decrease the abundance of type II sodium-dependent phosphate cotransporter Npt2a in the renal brush border membrane. Western blots showed that the protein abundance of Npt2a in XLKO renal brush border membrane was markedly increased (Figure 1, I and J). Immunofluorescence staining of kidney sections from P10 WT and XLKO mice also showed a pronounced increase in Npt2a staining in XLKO (Figure 1K), consistent with the hyperphosphatemia in XLKO pups.

Recombinant FGF23 injection rescues hyperphosphatemia in XLKO mice. To address whether the phenotype of XLKO mice resulted from reduced FGF23 levels, WT and XLKO mice were injected i.p. twice daily for 4 days with vehicle or full-length FGF23 harboring mutations responsible for autosomal dominant hypophosphatemic rickets $\left(\mathrm{FGF} 23^{\mathrm{R} 176 \mathrm{Q} / \mathrm{R} 179 \mathrm{Q}}\right)$; these mutations inhibit proteolytic inactivation of FGF23. Upon FGF23 ${ }^{\mathrm{R} 176 \mathrm{Q} / \mathrm{R} 179 \mathrm{Q}}$ administration, the serum phosphate levels declined significantly in both WT and XLKO mice, and the elevated phosphate levels in XLKO mice were normalized to the levels in vehicle-injected 
A

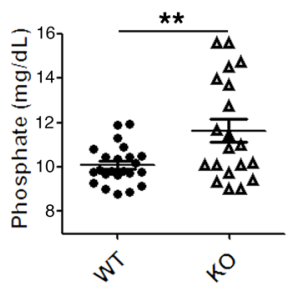

D

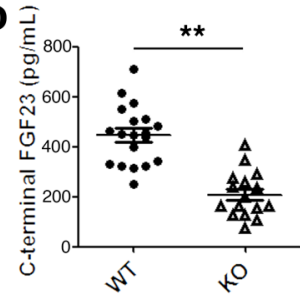

B

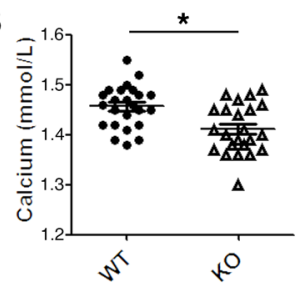

$\mathbf{E}$

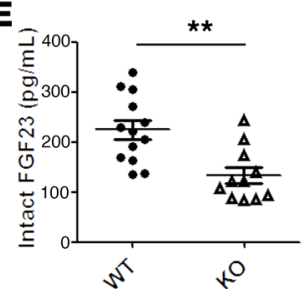

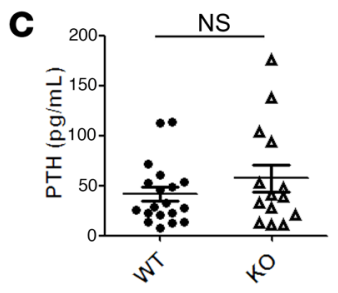

F

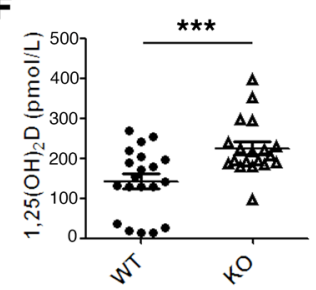

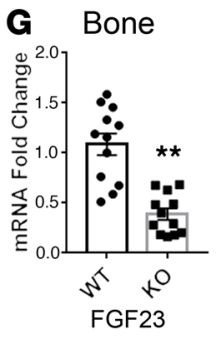

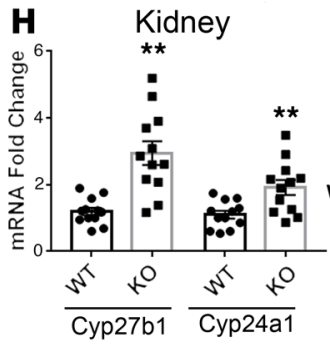

$\mathbf{K}$
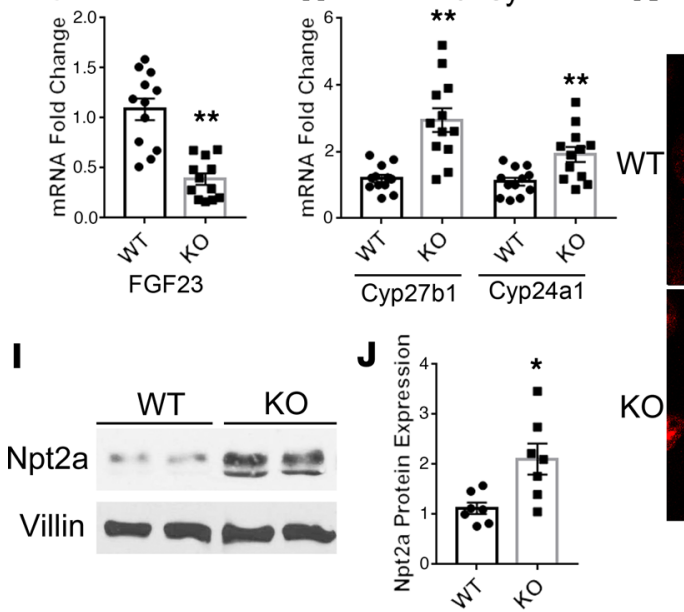

$$
\text { Npt2a }
$$
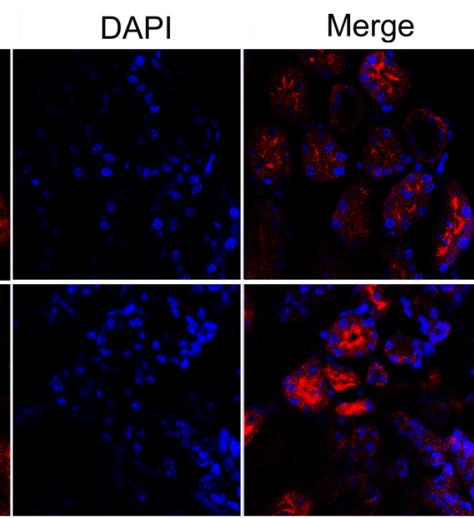

Figure 1. XLKO mice display decreased FGF23 levels with consequent alterations in phosphate and vitamin D metabolism. (A-F) Serum parameters of WT and KO mice at P10, including (A) phosphate, (B) blood ionized calcium, (C) PTH, (D) C-terminal FGF23, (E) intact FGF23, and (F) 1,25(OH) 2 D levels. (G) FGF23 mRNA levels in femurs and (H) Cyp27b1 and Cyp24a1 mRNA levels in renal tissue from P10 KO and WT mice. Data are shown as mean \pm SEM of littermates from 3 independent litters ( $n=12$ mice per group). (I) Western blot of Npt2a protein in renal brush border membrane samples from P10 KO and WT. Villin was used as a loading control. The blot is a representative of 3 independent experiments. (J) Densitometric quantification of renal brush border Npt2a protein from the Western blot experiments shown in panel I. (K) Npt2a and DAPI immunostaining of kidney sections from P10 XLKO and WT mice. ${ }^{*} P<0.05,{ }^{* *} P<0.01$, and NS, not significant, calculated by unpaired, 2-tailed Student's $t$ test.

WT mice (Figure 2A). The blood ionized calcium levels in WT mice were significantly decreased upon FGF23 injection. On the other hand, the calcium level was surprisingly elevated and corrected to normal in XLKO mice by FGF23 administration, suggesting that the decreased calcium level observed in XLKO mice may be secondary to the elevated phosphate level (Figure 2B). Moreover, WT mice displayed the expected decrease in Cyp27b1 mRNA and the increase in Cyp24a1 mRNA expression, as measured by qRT-PCR (Figure 2, C and D). On the other hand, there was a more dramatic response to FGF23 in XLKO mice. Cyp27b1 mRNA levels were repressed over 90\% in XLKO mice, and Cyp24a1 mRNA levels were induced by approximately 3.5 -fold (Figure 2, C and D), predicting a decline of the elevated $1,25(\mathrm{OH})_{2} \mathrm{D}$ levels in XLKO mice. These results demonstrated that the phosphate and vitamin D phenotype in XLKO mice results from FGF23 deficiency.

$X L \alpha$ ablation does not impair PTH-induced FGF23 production or CAMP generation but dampens IP3 and PKC levels in osteocytes. PTH stimulates FGF23 production via a Gsa/cAMP-mediated mechanism (15-17), and PTH1R has been shown to couple to XLas $(22,23)$. Injection with human PTH (aa 1-34) $(50 \mathrm{nmol} / \mathrm{kg})$ significantly augmented serum C-terminal FGF23 levels in both WT and XLKO pups after 2 hours, and the levels were comparable between the 2 genotypes (Figure 3A). Skeletal FGF23 
A
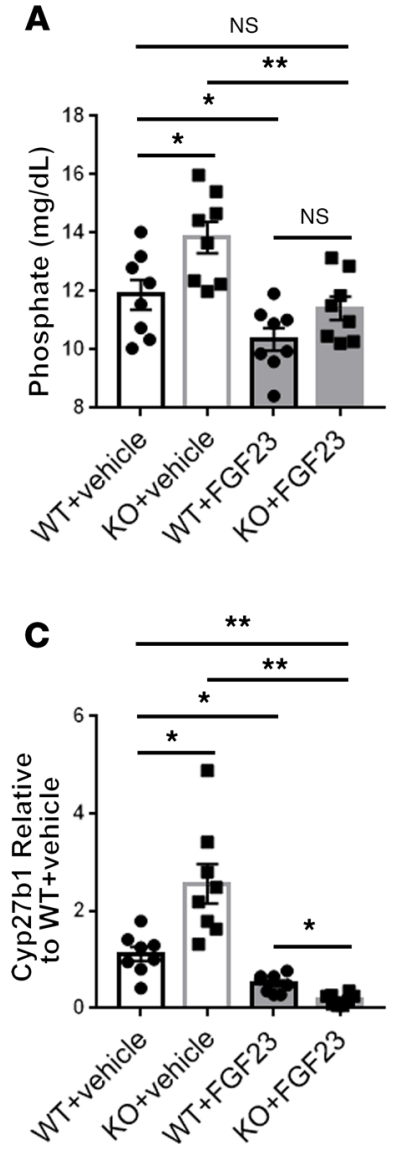
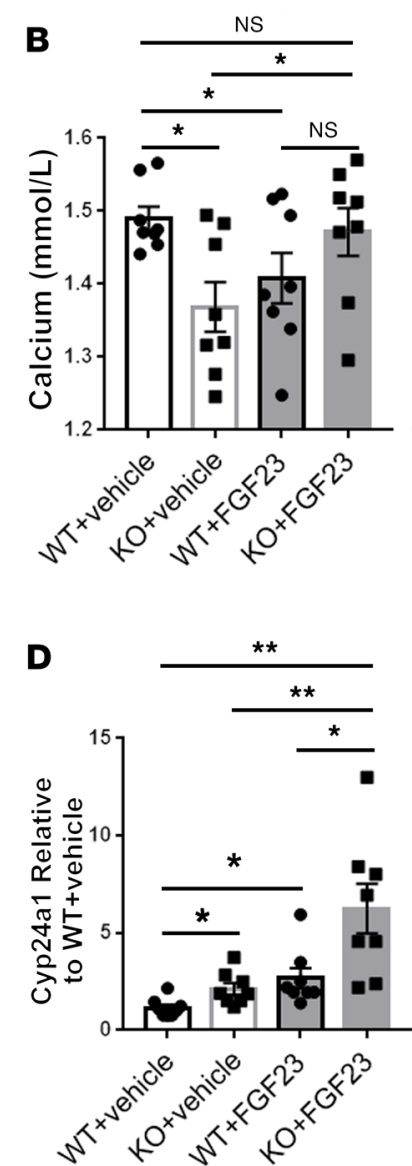

Figure 2. Injection of recombinant FGF23 rescues the disrupted mineral ion levels in XLKO mice. (A) Serum phosphate levels and (B) ionized calcium levels of WT and XLKO littermates injected with saline (vehicle) or a stable FGF23 analog for 4 days ( $n=9-14$ per group). (C and D) mRNA levels of (C) Cyp27b1 and (D) Cyp24a1 in kidneys from WT and KO pups injected with saline or FGF23 ( $n=8$ per group). Data are shown as mean \pm SEM. ${ }^{*} P<0.05 ;{ }^{* *} P<0.01$, and NS, calculated by 1-way ANOVA followed by Tukey's multiple-comparisons test.

mRNA expression was also significantly increased upon 2 hours of PTH treatment in both WT and XLKO mice, with no evidence of impaired PTH-induced FGF23 production in XLKO (Figure 3B). XLas, like Gas, can stimulate generation of the second messenger cAMP. We thus examined the cAMP levels in Ocy454 cells, an osteocyte-like cell line (37), in which we have previously ablated XLas by using CRISPR/Cas9 (38). Both the basal and PTH-induced levels of cAMP were higher in XLKO Ocy454 cells than in control cells (Supplemental Figure 2A). These enhanced cAMP levels in XLKO cells are consistent with our previous finding that Gs $\alpha$ protein level is moderately elevated in XLKO Ocy454 cells (38). We have previously demonstrated that ablation of XLas represses IP3 generation and the total levels of several PKC isoforms, including $\mathrm{PKC} \alpha$ and $\mathrm{PKC} \delta$, in renal proximal tubules (25). Similarly, the analysis of P10 XLKO femur samples revealed reduced levels of PKC $\alpha$ and PKC $\delta$ (Figure 3, C and D). As expected, XLas expression was undetectable in XLKO femurs, while Gsa expression appeared moderately increased (Figure 3C). Consistent with our in vivo results, FGF23 mRNA levels, as well as PKC $\alpha$ and PKC $\delta$ protein levels, were markedly reduced in XLKO Ocy454 cells (Figure 3, E-G). Moreover, the intracellular level of myo-inositol-1-phosphate (IP ${ }_{1}$ ), an indicator of IP3 generation (39), was also significantly repressed in XLKO cells (Supplemental Figure 2B).

PKC activation induces FGF23 production. Because IP3/PKC, but not cAMP, signaling was diminished upon XLas ablation, we then examined whether activation of PKC could rescue FGF23 expression in XLKO Ocy454 cells. Treatment with phorbol 12-myristate 13-acetate (PMA), a potent PKC activator, resulted in elevated FGF23 mRNA levels in both control (3.1-fold) and XLKO cells (7.3-fold), compared with vehicle-treated cells (Figure 4A). To further confirm the effect of PKC signaling on FGF23 production in vivo, we injected P9 WT and XLKO pups i.p. with saline (as control) or PMA (500 ng/g body weight) 
A

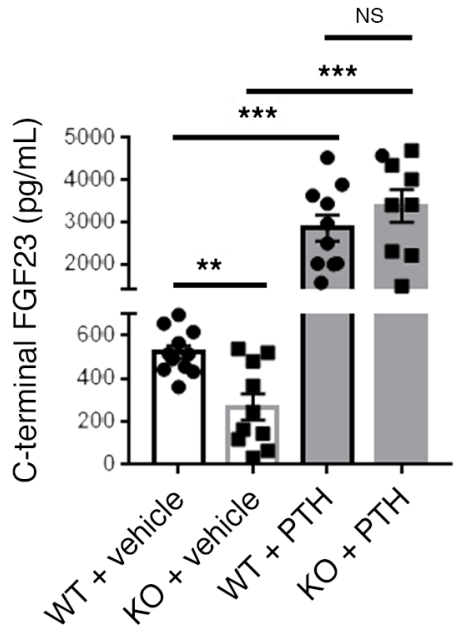

D

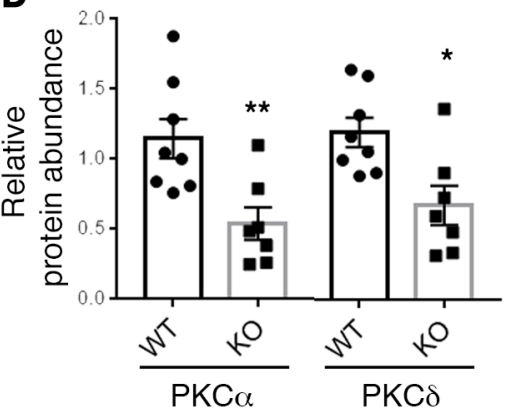

G

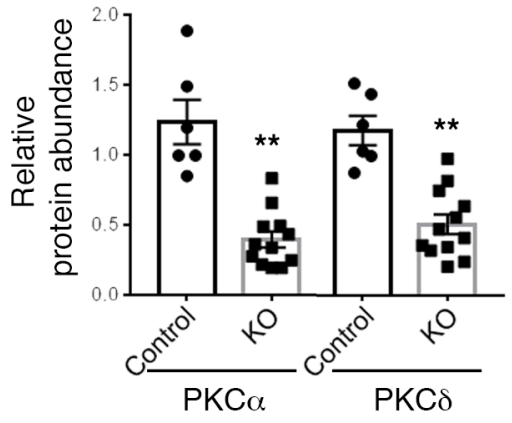

B

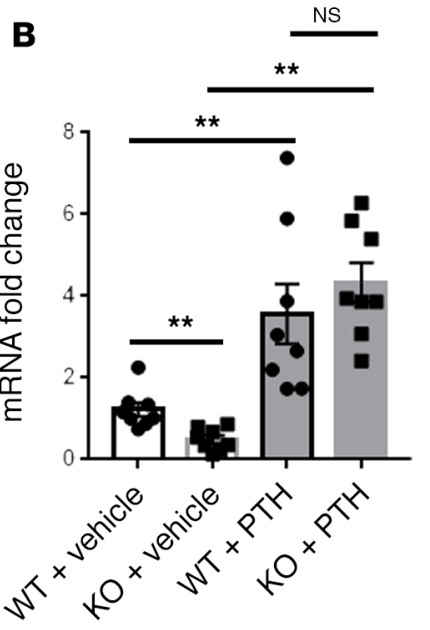

$\mathbf{E}$

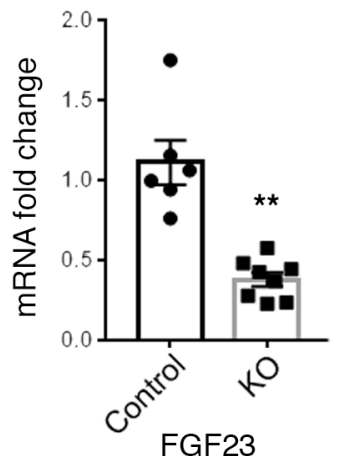

$\mathbf{F}$
C

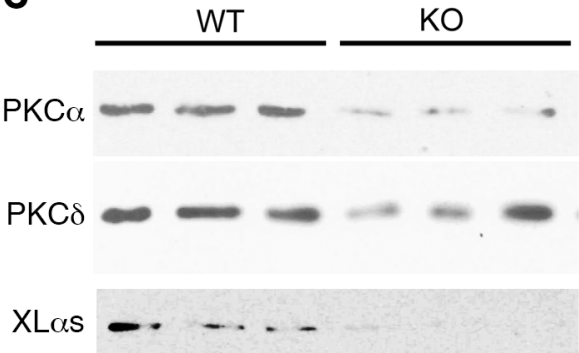

$\mathrm{Gs} \alpha$

Tubulin

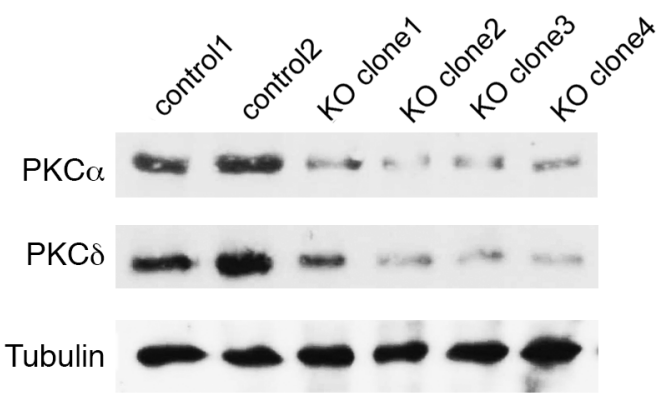

Figure 3. XLKO bones and XLKO cells show diminished PKC levels without impairment of the PTH/CAMP pathway. (A) Serum C-terminal FGF23 and (B) skeletal mRNA FGF23 levels upon 2-hour PTH treatment. ${ }^{* *} P<0.01$, ${ }^{* *} P<0.001$, and NS, calculated by Welch's $t$ test ( 2 tailed) followed by Bonferroni's correction. (C) Western blot analysis of PKC $\alpha$ and PKC $\delta$ using femur lysates from P10 WT and XLKO pups. Tubulin was used as a loading control. (D) Densitometric analysis of the relative abundance of PKC $\alpha$ and PKC $\delta$. Data are shown as mean \pm SEM of 7 or 8 mice from each group. ${ }^{*} P<0.05$, and ${ }^{* *} P<0.01$. (E) FGF23 mRNA expression in control and XLKO Ocy454 cells. (F) PKC $\alpha$ and PKC $\delta$ protein levels were detected by using Western blot in control and XLKO Ocy454 cells. (G) Densitometric analysis of data represented in $\mathbf{F}$ relative to tubulin. Data are shown as mean \pm SEM of 3 independent experiments. ${ }^{* *} P<0.01$. (D, E, and G) Significance between 2 groups was defined by 2 -tailed Student's $t$ test.

and analyzed the mice after 24 hours. Skeletal FGF23 mRNA levels, as well as serum FGF23 levels, were significantly increased in both WT and XLKO mice upon PMA injection, concurrently with a significant reduction in serum phosphate levels (Figure 4, B-D). Taken together, these results strongly suggested that activation of PKC stimulates FGF23 production.

Constitutively active G $\alpha q / P K C$ signaling stimulates FGF23 production. One of the most important stimulators of PKC is the $\mathrm{G}_{\mathrm{q} / 11}$ signaling pathway, which can be triggered by multiple GPCRs $(40,41)$. Because stimulation of PKC signaling by PMA stimulated FGF23 production in vitro and in vivo, we addressed whether activation of $\mathrm{G}_{\mathrm{q} / 11}$ signaling also leads to FGF23 production in osteoblasts and osteocytes. Rosa26-floxed stop-GNAQ ${ }^{\mathrm{Q} 209 \mathrm{~L}}\left(\mathrm{GNAQ}^{\mathrm{Q} 209 \mathrm{~L}}\right)$ mice (42), which drive expression of a constitutively active $\mathrm{Gq \alpha}$ mutant in cells that undergo Cre-mediated recombination, were crossed with osteocalcin-Cre-transgenic (Ocn-Cre-transgenic) mice, which express Cre recombinase in osteoblasts and their descendants. Serum C-terminal FGF23 levels, as well as skeletal FGF23 mRNA expression, were dramatically increased in P10 GNAQ ${ }^{\mathrm{Q} 209 \mathrm{~L}}$ Ocn-Cre mice (Figure 5, A and B), while the intact levels were only moderately increased (Figure 5C), indicating that the majority of overproduced FGF23 in the double-transgenic mice was coupled with increased FGF23 cleavage. 

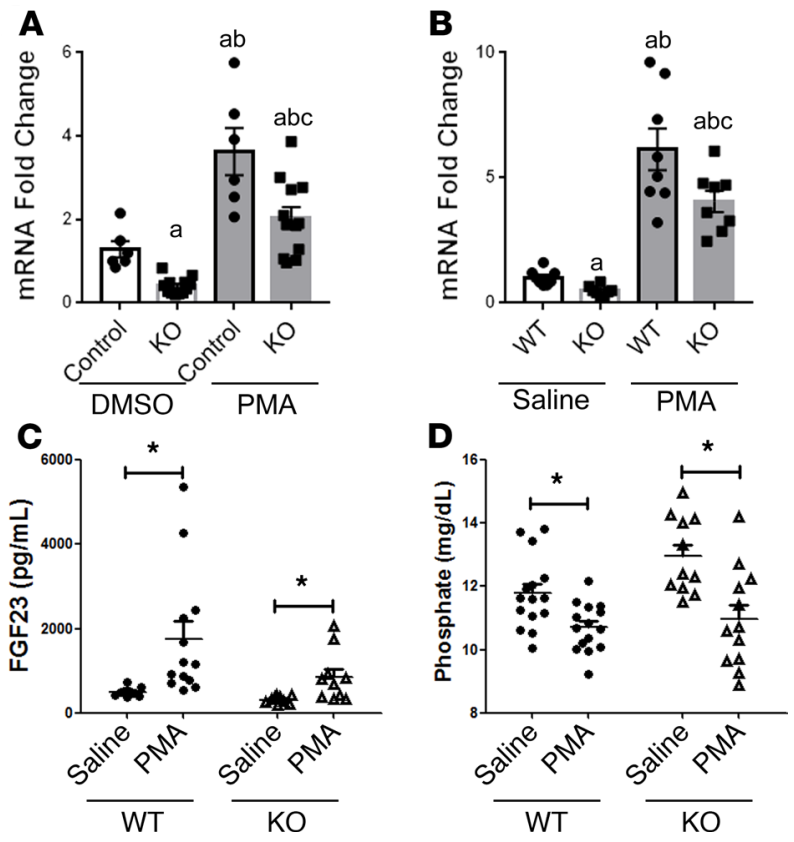

Figure 4. PKC activation stimulates FGF23 production and restores the FGF23 levels in XLKO. (A) Control and XLKO Ocy454 cells were treated with PMA or vehicle (DMSO) for 6 hours. mRNA levels of FGF23 were assessed by qRT-PCR. Data represent mean \pm SEM of values obtained from control and XLKO clones. ${ }^{a} P<0.05$ vs. DMSO-treated control cells; ${ }^{b} P<0.05$ vs. DMSO-treated KO cells; ${ }^{c} P<0.05$ vs. PMA-treated control cells. (B-D) WT and XLKO mice were injected i.p. with saline or PMA. (B) Femur FGF23 mRNA ( $n=8$ per group), (C) serum FGF23 (C-terminal assay; $n=11-15$ per group), and (D) serum phosphate ( $n=11-15$ per group) levels were examined 24 hours after the injection. ${ }^{a} P<0.05$ vs. saline-injected WT mice; ${ }^{b} P<$ 0.05 vs. saline-injected XLKO mice; ${ }^{c} P<0.05$ vs. PMA-injected WT mice. ${ }^{*} P<0.05$ vs. saline. Significance was determined by unpaired, 2-tailed Student's $t$ test.

In fact, phosphate levels were not changed in $\mathrm{GNAQ}^{\mathrm{Q} 209 \mathrm{~L}}$ Ocn-Cre mice (Figure 5D). We also crossed $\mathrm{GNAQ}^{\mathrm{Q} 209 \mathrm{~L}}$ with dentin matrix phosphoprotein 1-Cre (Dmp-Cre) mice, in which Cre is expressed in mature osteoblasts and osteocytes, and found a similar but milder increase of FGF23 production in GNAQ ${ }^{\mathrm{Q} 2095}$ Dmp1-Cre mice (Figure 5, E-G); however, phosphate remained unchanged between control and double-transgenic mice (Figure 5H). We also analyzed serum C-terminal and skeletal FGF23 mRNA levels in 2-month-old control and GNAQ ${ }^{\mathrm{Q} 209 \mathrm{~L}} \mathrm{Dmp1}$-Cre mice and found that FGF23 production remained elevated in double-transgenic mice (Supplemental Figure 3, A and B). Taken together, these results strongly suggested that $\mathrm{G}_{\mathrm{q} / 11}$ signaling is able to promote $\mathrm{FGF} 23$ production.

Because adult XLKO mice, unlike their early postnatal counterparts, displayed only a modest reduction of FGF23 levels, and because constitutively active $G_{q / 11}$ signaling promoted FGF23 synthesis both early postnatally and in adulthood, we compared mRNA levels of XL $\alpha$ s, as well as Gq $\alpha$ and G11 $\alpha$, in P10 and 8-week-old mouse femurs. qRT-PCR experiments revealed that the level of XLas in 8-week-old femur was only $1.8 \% \pm 0.9 \%$ of that in P10 femur, suggesting a dramatic decline of XLas expression with age. In contrast, the levels of Gq $\alpha$ and G11 $\alpha$ at 8 weeks were $63.1 \% \pm 1.8 \%$ and $75.3 \% \pm 2.4 \%$ of the levels at P10, respectively (Supplemental Figure 3C).

The $G_{q / 11} / P K C$ signaling-induced FGF23 production is dependent on MAPK signaling. Both control and XLKO Ocy454 clonal cells transduced with adenovirus encoding XLas showed marked elevation of FGF23 mRNA levels compared with cells transduced with adenoviral YFP used as control (Figure 6A, Supplemental Figure 4A). In addition, treatment of these cells individually with isozyme nonselective PKC inhibitors (bisindolylmaleimide I [Bisindol], Ro-31-8220, or Ro-32-0432) significantly, albeit incompletely, blocked the XLas overexpression-induced FGF23 production, demonstrating that XLas regulates FGF23 production via PKC signaling (Figure 6B). The rise of FGF23 mRNA levels by PMA treatment was completely blocked by treatment with these PKC inhibitors (Figure 6C). Similar results were obtained with Saos-2 cells, a human osteosarcoma cell line (Supplemental Figure 4B). PMA also stimulates production of inflammation mediators, such as IL-6, which has been recently shown to 

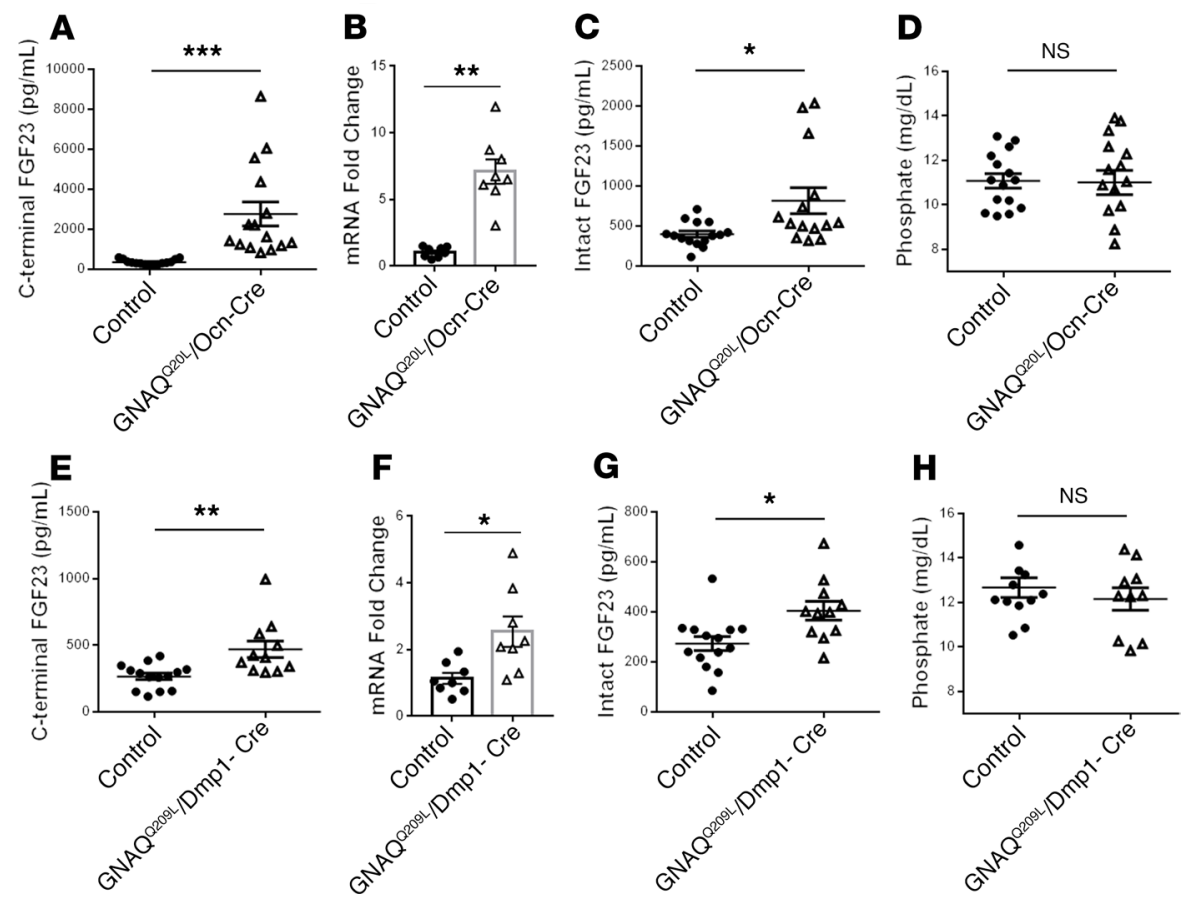

Figure 5. Constitutively active Gq $\alpha$ signaling induces FGF23 production in mice. (A) Serum C-terminal FGF23, (B) skeletal FGF23 mRNA, (C) intact FGF23, and (D) phosphate levels in GNAQ209L Ocn-Cre mice and control littermates. (E) Serum C-terminal FGF23, (F) skeletal FGF23 mRNA, (G) intact FGF23, and (H) phosphate levels in GNAQ ${ }^{\text {Q209L }}$ Dmp1-Cre mice and control littermates. ${ }^{*} P<0.05,{ }^{* *} P<0.01$, ${ }^{* *} P<0.001$, and NS, calculated by 2 -tailed Student's $t$ test.

directly stimulate FGF23 production (43). PMA treatment of Ocy454 cells significantly increased IL-6 mRNA levels, and 2 of the PKC inhibitors, Bisindol and Ro-32-0432, repressed PMA-stimulated IL-6 induction (Figure 6D). However, Ro-31-8220, which could inhibit both PMA- and XLas overexpression-induced FGF23 production, further increased the PMA-induced IL-6 mRNA level rather than repressing it (Figure 6D), strongly suggesting that PKC activation-mediated FGF23 production was not associated with IL-6 expression. The level of ERK1/2 phosphorylation (p-ERK1/2) was also dramatically repressed in XLKO bones and in XLKO Ocy454 cells (Figure 6, E-H). PMA treatment also stimulated p-ERK1/2 levels markedly in control and XLKO Ocy454 cells (Figure 6, I and J). In addition, MEK inhibitor U0126 inhibited PMA-induced FGF23 elevation, suggesting that MAPK signaling is downstream of PKC activation and plays an important role in mediating PKC activation-mediated FGF23 production (Figure 6K). Because FGFR1 signaling is a known mediator of FGF23 production in bone, we also examined FGFR1 expression in P10 femurs and clonal Ocy454 cells and revealed significantly reduced FGFR1 mRNA levels in XLKO bones and cells (Supplemental Figure 5, A and B). We then induced FGFR1 transcription in Ocy454 cells through the CRISPR/Cas9 synergistic activation mediator (44), using several activator sgRNAs. Two of the FGFR1 promoter-targeting sgRNAs (activator 1 and activator 2) resulted in significantly increased FGFR1 mRNA expression in WT and XLKO cells (Supplemental Figure 5C). These sgRNAs also raised FGF23 mRNA levels in both WT and XLKO cells and fully rescued the reduced FGF23 levels in XLKO cells (Supplemental Figure 5D). Nevertheless, FGFR1 mRNA expression levels were not altered in femurs from GNAQ ${ }^{2209 \mathrm{~L}} \mathrm{Ocn}$-Cre or GNAQ $^{\mathrm{Q} 209 \mathrm{~L}}$ Dmp1-Cre double-mutant mice (Supplemental Figure 5, E and F), which, as shown above, displayed elevated serum and skeletal mRNA levels of FGF23. These results suggested that the $\mathrm{G}_{\mathrm{q} / 11} /$ PKC activation-induced FGF23 synthesis may not involve elevation of FGFR1 levels.

Skeletal PKC activity and MAPK signaling are elevated in a mouse model of XLH. XLH is the most common form of inherited rickets, which is driven by hypophosphatemia caused by excess FGF23 production (45). Hyp mice, a murine model of XLH, also have dramatically elevated FGF23 levels. To assess the level of overall PKC activity in Hyp mice bones, we used Western blot analysis using an antibody recognizing proteins phosphorylated at PKC consensus sites. Our results showed significantly increased 

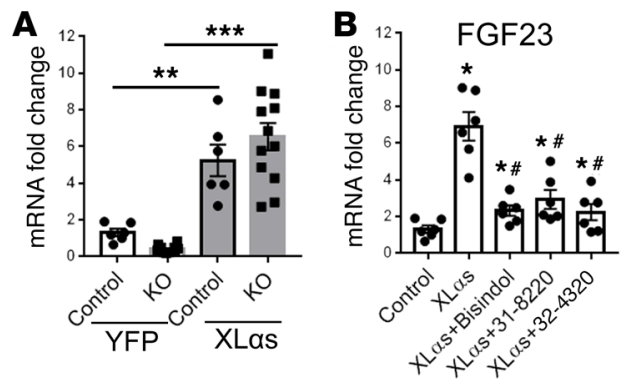

$\mathbf{E}$

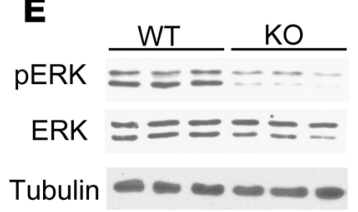

$\mathbf{F}$
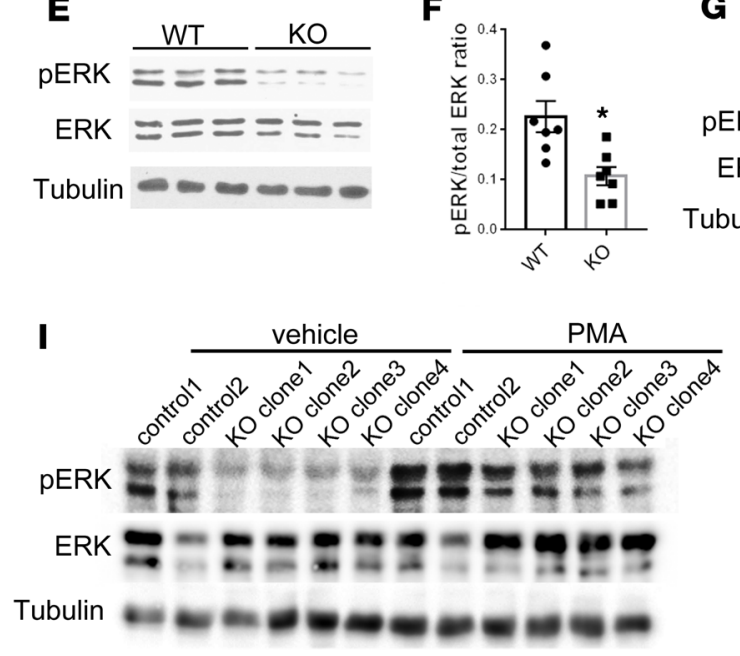
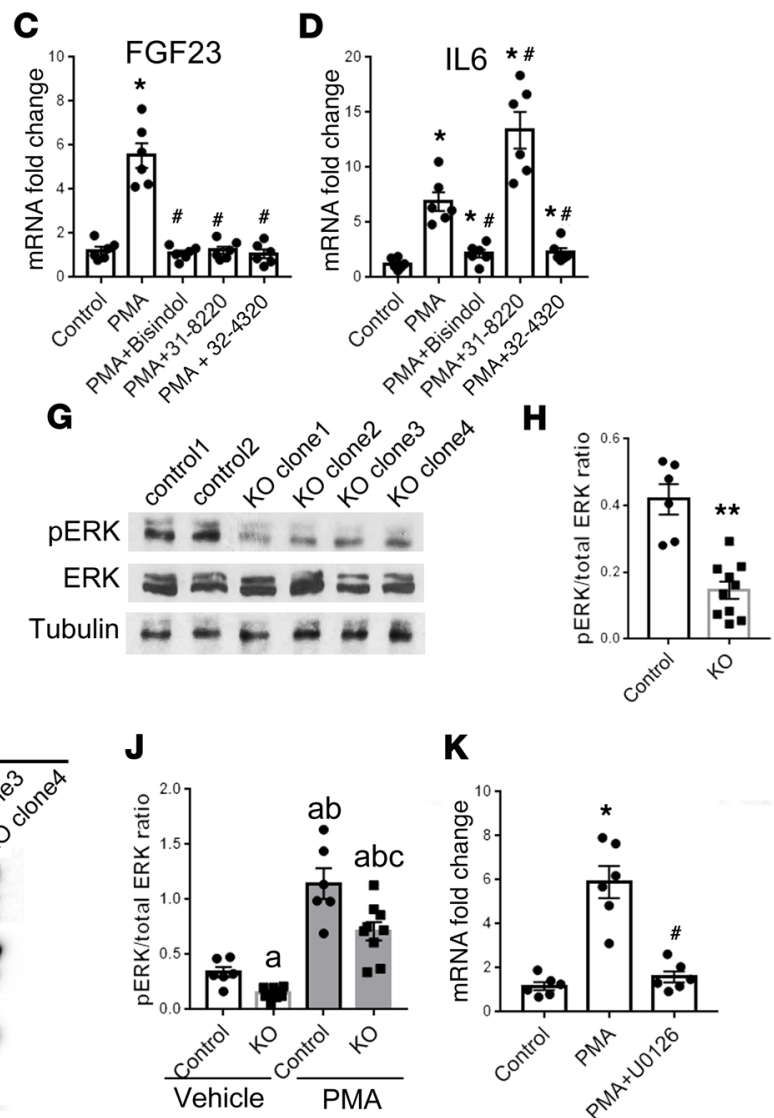

$\mathbf{K}$

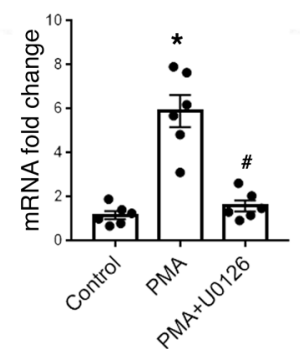

Figure 6. PKC activation mediates FGF23 production by promoting MAPK signaling. (A) FGF23 mRNA expression in YFP- and XLas-overexpressing Ocy454 cells. ${ }^{* *} P<0.01$, and ${ }^{* *} P<0.001$, determined by Welch's $t$ test ( 2 tailed) followed by Bonferroni's correction. (B) FGF23 mRNA expression in control or XLas adenovirus-infected Ocy454 cells treated with PKC inhibitors, bisindolylmaleimide I (Bisindol), Ro-31-8220, or Ro-32-0432. Data are shown as mean \pm SEM of 3 independent experiments. ${ }^{*} P<0.05$ vs. control group; ${ }^{*} P<0.05$ vs. XLas-overexpressing Ocy 454 cells. (C and $\left.\mathbf{D}\right)$ Ocy454 cells were treated with PMA, with PKC inhibitors. qRT-PCR analysis of (C) FGF23 and (D) IL-6 mRNA after 6 hours of treatment. Data are shown as mean \pm SEM of 4 independent experiments. ${ }^{*} P<0.05$ vs. control group; ${ }^{P} P<0.05$ vs. PMA-treated group. (B-D) Significance was calculated by 1 -way ANOVA followed by Tukey's multiple-comparisons test. (E) Representative Western blot of phosphorylated ERK1/2 (p-ERK) and total (phosphorylated and nonphosphorylated) ERK1/2 (total ERK) in femur bone lysate samples from P10 XLKO (KO) and WT mice. Loading control: tubulin. (F) Densitometric analysis of the ratio of p-ERK to total ERK in KO and WT femurs. Data are shown as mean \pm SEM of 7 mice from each group. (C) Western blot analysis of $p$-ERK, ERK, and tubulin levels in control and XLKO Ocy454 cells. (H) Densitometric analysis of $p$-ERK/total ERK ratio represented in $\mathbf{G}$. Data are shown as mean \pm SEM from 3 independent experiments. $(\mathbf{F}$ and $\mathbf{H}){ }^{*} P<0.05,{ }^{* *} P<0.01$ by 2 -tailed Student's $t$ test. (I) Western blot analysis of $p$-ERK, ERK, and tubulin in XLKO and control cells treated with PMA or vehicle. (J) Densitometric analysis of $p$-ERK/ERK ratio represented in I. a $P<0.05$ vs. vehicle-treated control cells; ${ }^{b} P<0.05$ vs. vehicle-treated KO cells; ${ }^{\mathrm{C}} P<0.05$ vs. PMA-treated control cells. (K) FGF23 mRNA levels in Ocy454 cells treated with PMA, with or without MEK inhibitor U0126. Data are shown as mean \pm SEM of 3 independent experiments. ${ }^{*} P<0.05$ vs. control group; ${ }^{\#} P<0.05$ vs. PMA-treated group. Statistical differences in $\mathbf{J}$ and $\mathbf{K}$ were assessed with 1-way ANOVA followed by Tukey's test.

levels of some of these PKC-phosphorylated proteins in Hyp bones (Figure 7, A and B), consistent with enhanced PKC activity. The level of p-ERK1/2 in Hyp bones was also significantly increased (Figure 7, A and C). A single injection of P10 WT and Hyp littermates with the PKC inhibitor Ro-32-0432 revealed, 24 hours later, a mild trend toward lower serum FGF23 and higher serum phosphate levels in Hyp mice compared with vehicle-injected mice (Supplemental Figure 6, A and B). Because ablation of XLas reduced PKC activation and FGF23 production in vivo, we then intercrossed XLKO and Hyp mice. At P10, XLKO/Hyp double-mutant mice were still hypophosphatemic and displayed increased FGF23 levels compared with WT mice. However, compared with Hyp mice, the XLKO/Hyp double mutants showed a moderate but significant decrease in C-terminal FGF23 levels, as well as intact FGF23 levels (Figure 7, D and E). Likewise, serum phosphate levels were significantly increased in the XLKO/Hyp double mutants compared with Hyp littermates (Figure 7F). These results suggested that suppression of XLas/PKC signaling could alleviate the biochemical phenotype of Hyp mice. 


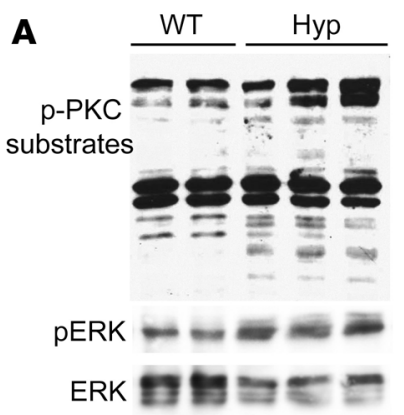

B

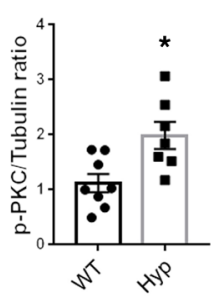

Tubulin
D

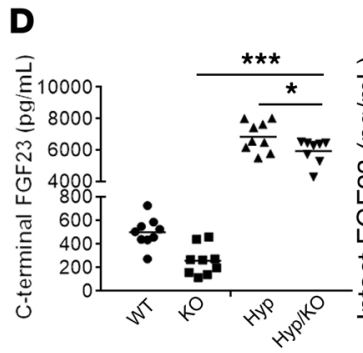

E
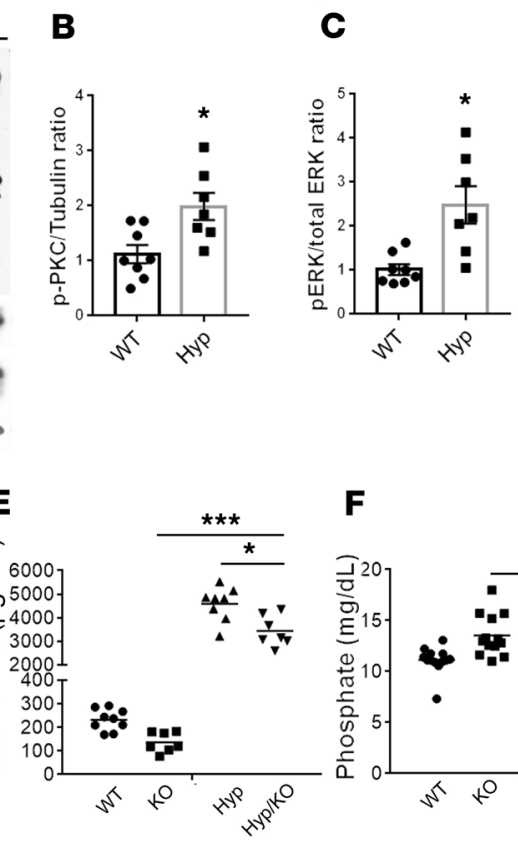

$\mathbf{F}$

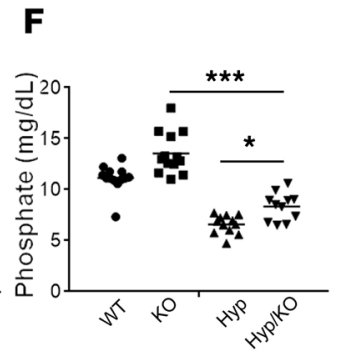

Figure 7. Elevated PKC activity contributes to the elevated FGF23 in Hyp mice. (A) Western blot analysis of phosphorylated PKC (p-PKC) substrates, p-ERK, and total ERK in WT and Hyp mice. (B) Densitometric analysis of the relative abundance of p-PKC substrates. (C) Densitometric analysis of $p$-ERK/total ERK ratio represented in A. Data are shown as mean \pm SEM of 7 or 8 mice from each group. ${ }^{*} P<0.05$, calculated by unpaired, 2 -tailed Student's $t$ test. (D) Serum C-terminal FGF23, (E) intact FGF23, and (F) phosphate levels in Hyp and XLKO intercross littermates. ${ }^{*} P<0.05$, and ${ }^{* * *} P<0.001$, determined by Welch's $t$ test ( 2 tailed) followed by Bonferroni's correction.

\section{Discussion}

Our study shows that the unique Ga subunit XLas, which, in spite of being mostly identical to Gs $\alpha$, promotes IP3/PKC signaling, is required for FGF23 production in early postnatal mice. Our investigations also revealed that the IP3/PKC signaling pathway, presumably downstream of an as-yet-unidentified GPCR, is an important mediator of skeletal FGF23 production through a mechanism dependent on MAPK signaling (Figure 8).

Excess FGF23 production was observed in response to the activation of PKC, stimulated by XLas overexpression, phorbol esters, or constitutive $\mathrm{G}_{\mathrm{q} / 11}$ activation. PKC activation has been shown to promote MAPK signaling in osteoblastic cells (46). We also observed elevated ERK1/2 phosphorylation upon PMA treatment of Ocy454 cells, and the baseline level of ERK1/2 phosphorylation was diminished in XLKO bones, which concurrently had reduced PKC $\alpha$ and PKC $\delta$ levels. Taken together with the finding that MAPK inhibitor U0126 blocked the PKC activation-induced FGF23 production, it appears that PKC mediates FGF23 production by promoting, at least partly, the MAPK signaling pathway. In contrast, our findings from PKC inhibitor experiments strongly suggest that PKC-mediated FGF23 production is independent of IL-6.

PKC inhibitors blunted the effect of XLas overexpression but entirely abrogated the effect of PMA treatment on FGF23 mRNA levels in Ocy454 cells. This finding may reflect the possibility that the XLas overexpression caused a much stronger PKC activation than did the PMA treatment. Alternatively, XLas overexpression may have promoted FGF23 production additionally through the cAMP signaling pathway because it can result in significantly increased basal cAMP production $(47,48)$. However, we found that PMA treatment did not fully restore the levels of ERK1/2 phosphorylation in XLKO cells, suggesting that the action of XLas entails additional pathways that may converge on MAPK signaling. One of those may be FGFR signaling. Fgfr1 mRNA levels were diminished in XLKO bones, and overexpression of Fgfr1, which presumably enhanced MAPK signaling, could override the suppressed FGF23 synthesis caused by XLas deficiency. This finding may suggest that the presumably diminished FGFR1 levels contribute to the low FGF23 levels in XLKO cells. Future studies are needed to fully understand the Gq/IP3/PKC pathway-independent role of XL $\alpha$ s in the regulation of FGFR1/ MAPK signaling and FGF23 synthesis. Nonetheless, it is important to note that our mice in which Gq/ PKC signaling was constitutively activated in bone showed a pronounced FGF23 overproduction in the 


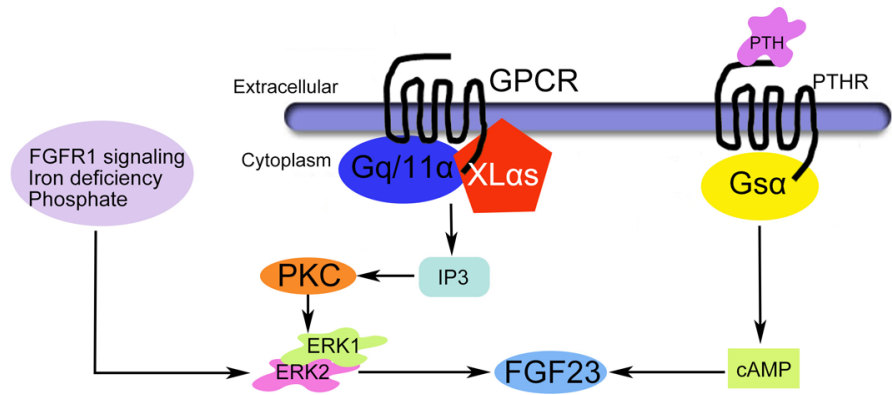

Figure 8. A model of XLas function in FGF23 production. In bone cells, $X L \alpha s$ and $G_{q / 11}$ a promote FGF23 production, presumably in response to the activation of an as-yet-unknown GPCR. XLas or $G_{q / 11} \alpha$ activates PKC and stimulates the MAPK/ERK1/2 pathway, which has also been implicated in the FGF23 production regulated by FGFR1 signaling, iron deficiency, and phosphate. The action of PTH on FGF23 synthesis involves Gs $\alpha /$ CAMP signaling and appears to be independent from the pathway mediated by $\mathrm{G}$ protein-IP3/PKC.

absence of elevated Fgfr1 expression. Thus, it appears that the IP3/PKC signaling-mediated FGF23 production does not require elevation of FGFR1 levels.

FGF23 increases urinary phosphate excretion by repressing the activity of NaPi-2a and NaPi-2c cotransporters to reduce renal phosphate reabsorption to maintain systemic phosphate homeostasis. In mice expressing the constitutively active Gq $\alpha$ mutant in bone cells, serum phosphate levels were not altered, despite the modestly increased levels of serum intact FGF23 in the mutant mice. It is possible that the urinary phosphate excretion was increased in those mice, but our assay was unable to detect the change in serum phosphate. In contrast, the PMA-injected mice displayed low serum phosphate compared with vehicle-injected mice. The latter finding may reflect a direct effect of PMA on the renal proximal tubules because PKC activation promotes renal phosphate excretion (49).

We detected increased levels of phosphorylated proteins by PKC in Hyp bones, suggesting elevated PKC activity in this mouse model of XLH. Consistent with our finding, a microarray analysis of Hyp bones revealed 41 genes upregulated by more than 2 -fold (50), and a number of them are positively regulated by PKC activation in bone or in other cell types, including Egr2, Eln, Prkg2, Cyp11a1, Lipg, Gem, and F3 (51-54). The underlying cause of elevated skeletal PKC activity in XLH is currently unknown. Nonetheless, our results obtained from intercrosses between Hyp and XLKO, as well as from the injection of a PKC inhibitor into Hyp and control mice, suggest that the increased PKC activation in XLH contributes to the elevated FGF23 production.

Based on our PTH injection experiments, the novel FGF23 synthesis mechanism involving G protein/PKC activation does not mediate the action of PTH. This is consistent with previous studies showing that PTH-induced FGF23 production occurs via cAMP-mediated mechanisms $(15-18,55)$. In contrast, $1,25(\mathrm{OH})_{2} \mathrm{D}$ - and phosphate-induced FGF23 production may depend, at least partly, on the $\mathrm{G}$ protein/PKC pathway, given that the significantly elevated $1,25(\mathrm{OH})_{2} \mathrm{D}$ and phosphate levels in XLKO pups were insufficient to maintain the normal level of FGF23. Likewise, XLKO pups have been reported to have serum iron deficiency (38), and therefore, it appears that lack of XLas also impedes the effect of iron deficiency on FGF23 production. Consistent with these conclusions, MAPK signaling has been implicated in FGF23 synthesis in response to both iron deficiency and phosphate (56-58). Nonetheless, further studies are needed to address whether XLas or IP3/PKC signaling modulates the actions of these major FGF23 regulators.

Adult XLKO mice displayed only mildly reduced serum FGF23 levels as opposed to P10 XLKO, which had significantly decreased FGF23 production. The outcome of XLas ablation on FGF23 levels may be masked by the systemic alterations previously documented in adult XLKO mice, such as increased levels of circulating catecholamines (59). It is also possible, however, that XLas is not expressed at significant levels in adult osteocytes/osteoblasts and, therefore, has a minor role in FGF23 synthesis. $\mathrm{XL}$ as protein or mRNA is readily detectable in adult mouse bone and adult human bone marrow stromal cells $(26,28)$, but we found a dramatic decline of XLas mRNA levels from P10 to age 8 weeks in mouse femurs, supporting the latter possibility. In contrast, the levels of Gq $\alpha$ or G11 $\alpha$ did not change as dramatically with age, making it plausible that the Gq family of $\alpha$ subunits take over XLas's role as a mediator of FGF23 production in adulthood. 
Genetic mutations affecting the paternal GNAS allele result in complete loss of XLas in most tissues because of the genomic imprinting of its promoter. Thus, our findings predict that children with paternal GNAS disruption, i.e., pseudo-pseudohypoparathyroidism, can have reduced FGF23 production and resultant hyperphosphatemia. Accordingly, certain pediatric patients carrying such genetic defects have been reported to have normal or elevated serum phosphate with mildly elevated PTH levels (29-35). However, it should be noted that patients with paternal GNAS mutations also have Gs $\alpha$ haploinsufficiency, which may contribute to those findings or could mask the phenotypic expression of XLas deficiency alone.

In summary, we discovered a potentially novel mechanism of FGF23 synthesis involving an as-yet-unidentified GPCR that signals via the IP3/PKC pathway feeding into MAPK signaling (Figure 8). Further elucidation of the underlying mechanisms may identify novel drug targets for treating the diseases caused or affected by dysregulated FGF23 production. As such, it will be important to identify the GPCR(s) that couples to XL $\alpha$ s and/or $\mathrm{G}_{\mathrm{q} / 11} \alpha$ in this context. Targeting that receptor by an agonist or an antagonist may allow FGF23 levels to be fine-tuned as needed.

\section{Methods}

Mice. XLKO mice were generated by disrupting the first exon of XLas on the paternal allele, thus resulting in the global ablation of this protein (60). These mice were maintained on a CD1 genetic background.

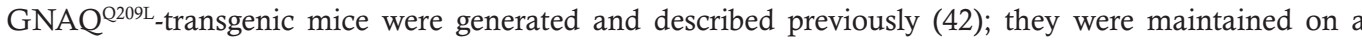
C57BL/ 6 background. Ocn-Cre, Dmp1-Cre, and Hyp mice were also on a C57BL/6 background and purchased from The Jackson Laboratory. Both male and female Hyp mice were included in the analyses, which were performed on P10.

Chemical compounds. Recombinant human FGF23 containing naturally occurring pathogenic Arg-toGln mutations at its ${ }^{176} \mathrm{RHTR}^{179}$ proteolytic cleavage site, FGF23 ${ }^{\mathrm{R} 176 \mathrm{Q} / \mathrm{R} 179 \mathrm{Q}}$ (aa 25-251), was generated in-house (61). Synthetic human PTH (aa 1-34) was synthesized by The Peptide/Protein Core Facility at Massachusetts General Hospital. The broad-range PKC inhibitor Bisindol was purchased from MilliporeSigma; another 2 PKC inhibitors, Ro-32-0432 and Ro-31-8220, were from Santa Cruz Biotechnology. MEK inhibitor U0126 was purchased from Cell Signaling Technology. PMA, forskolin, and all other reagents were purchased from MilliporeSigma.

Measurement of serum biochemistries. Serum concentrations of phosphate, $\mathrm{Ca}^{2+}, \mathrm{PTH}$, and $1,25(\mathrm{OH})_{2} \mathrm{D}$ were examined by using blood samples obtained from the carotid artery, as described previously (24). C-terminal and intact FGF23 concentrations were measured with the Mouse/Rat FGF-23 (C-Term) and Mouse/Rat FGF-23 (Intact) ELISA kits (Quidel).

Cell culture. The Ocy454 cells, which were previously described (37), were maintained in $\alpha$-minimum essential medium ( $\alpha$-MEM, Gibco) with $10 \% \mathrm{FBS}$ at $33^{\circ} \mathrm{C}$ and differentiated into osteocyte-like cells by growing at $37^{\circ} \mathrm{C}$ for 1 week $(37,62)$. XLKO and control clonal Ocy 454 cells were generated previously (38) and used here for protein and mRNA analyses. Saos-2 cells were obtained from ATCC (ATCC HTB-85) and cultured at $37^{\circ} \mathrm{C}$ in McCoy's 5a medium (Gibco) supplemented with $15 \%$ FBS. To test the effects of PMA and different inhibitors, the cell culture was changed to $\alpha$-MEM with $2 \%$ FBS overnight before treating with $1 \mu \mathrm{M}$ PMA, with or without inhibitor $(10 \mu \mathrm{M}$ Bisindol, $10 \mu \mathrm{M}$ Ro-32-0432, $10 \mu \mathrm{M}$ Ro-31-8220, or $10 \mu \mathrm{M}$ U0126) for 6 hours.

Generation of FGFR1 transcriptionally activated Ocy454 cells by CRISPR/sgRNA-directed synergistic activation mediator. Four sgRNAs targeting the Fgfr1 gene were designed through the Synergistic Activation Mediator Cas9 activator design tool (http://sam.genome-engineering.org/database/) and subcloned into the lenti sgRNA(MS2)_zeo vector (Addgene). Based on the efficiency of Fgfr1 activation, 2 activation guides, FGFR1 activator 1 and activator 2, were selected for further analysis. Viral packaging was performed in 293T cells (ATCC) using standard protocols by cotransfection of the lentiviral plasmid along with psPAX2 (Addgene) and MD2.G (Addgene) using PolyJet DNA Transfection Reagent (SignaGen Laboratories). XLKO and control Ocy454 cells were transduced with lentiviral dCas9-VP64, MS2-P65-HSF1, together with lentiviral FGFR1 activator 1, activator 2, or sgRNA(MS2)_zeo, and then selected with blasticidin (5 $\mu \mathrm{g} / \mathrm{mL})$, hygromycin $(100 \mu \mathrm{g} / \mathrm{mL})$, and zeocin $(200 \mu \mathrm{g} / \mathrm{mL})$ to enrich for cells stably expressing the lentivirus. Cells were maintained in the selection medium throughout the duration of the experiment.

cDNA synthesis and qRT-PCR analysis. Total RNA isolated from kidneys and femurs of P10 mice, as well as that from Ocy454 and Saos-2 cells, was prepared with the RNeasy Plus Mini Kit (Qiagen), and cDNA was synthesized with the ProtoScript II First Strand cDNA Synthesis Kit (New England BioLabs), as 
previously described (63). qRT-PCR analysis on FGF23 was tested by using FGF23-FAM and Actb-VIC TaqMan primers and TaqMan Fast Advanced Master Mix (Applied Biosystems). qRT-PCR analysis on all other genes was performed with specific primers (Supplemental Table 1) and PowerUp SYBR Green Master Mix (Applied Biosystems) with Actb as a reference gene.

Cell/tissue lysis and Western blot analysis. Cells and femur tissues were lysed by using HNTG lysis buffer (20 mm HEPES, pH 7.4; 150 mm NaCl; 10\% glycerol; 1\% Triton X-100, 1.5 mm $\mathrm{MgCl}_{2} ; 1.0 \mathrm{~mm}$ EGTA) containing Complete Protease inhibitor mixture tablets (Roche). Lysates were resolved by $10 \%$ SDS-PAGE and transferred to nitrocellulose membranes (Bio-Rad). Western blots were then incubated with specific antibodies. Antibody against p-ERK was purchased from MilliporeSigma (catalog M8159) and tubulin antibody from Abcam (catalog ab6046). Antibodies against phospho-PKC substrates (catalog 2261S), total ERK (catalog 9102S), PKC $\alpha$ (catalog 9960S), and PKC $\delta$ (catalog 9960S) were from Cell Signaling Technology. Gs $\alpha$ antibody, which recognizes both Gs $\alpha$ and XL $\alpha$, was purchased from MilliporeSigma (catalog 06-237).

Histology, immunohistochemistry, and immunofluorescence staining. Kidney sections from P10 XLKO and WT mice were stained with Npt2a antibody (64) at $4^{\circ} \mathrm{C}$ overnight and then stained with Alexa Fluor 568conjugated donkey anti-rabbit antibody (Life Technologies) at room temperature for 1 hour. Immunoreactivity was visualized and analyzed with a Nikon Eclipse Ni microscope and SPOT Microscope Imaging Software or with a Zeiss LSM 510 confocal microscope and Zeiss Zen software.

Isolation of renal brush border membranes. Brush border membrane portions were prepared from the kidneys of P10 WT and XLKO mice as described previously (65) with minor modifications. Isolated brush border membrane proteins were lysed in a Tris-buffered solution containing $150 \mathrm{mM} \mathrm{NaCl}$ and $1 \%$ Triton $\mathrm{X}-100$. Measurement of protein concentrations in the samples with antibodies specific for Npt2a (64) and Villin (Santa Cruz Biotechnology, sc-58897) were performed as described previously (24).

$I P_{1}$ and $c A M P$ assays. The amount of $\mathrm{IP}_{1}$, a downstream metabolite of IP3, in control and XLKO Ocy 454 cells was tested using the IP ${ }_{1}$ HTRF assay kit (Cisbio) as previously described (25). For cAMP assay, confluent control and XLKO Ocy454 cells in 96-well plates were treated with different concentrations of PTH, forskolin, or vehicle, with $2 \mathrm{mM}$ 3-isobutyl-1-methylxanthine for 30 minutes at room temperature, before lysing the cells for RIA measurement.

Recombinant FGF23 ${ }^{R 176 / R 179 Q}, P T H, P M A$, and Ro-32-0432 injections. The recombinant FGF23 compound $(61,66)$ or vehicle was injected i.p. into WT and XLKO mice (250 ng/g body weight) from P6 to $\mathrm{P} 10$ at 12-hour intervals (67). The mice were sacrificed 12 hours after the last injection, and blood and kidneys were collected for analysis. To test PTH-induced FGF23 production in WT and XLKO mice, mice were injected subcutaneously with either PTH (aa 1-34) $(50 \mathrm{nmol} / \mathrm{kg}$ ) or vehicle (10 mm citric acid, $150 \mathrm{~mm} \mathrm{NaCl}, 0.05 \%$ Tween 80, pH 5.0) for 2 hours before analysis (55). To test the effect of PMA on FGF23 production, WT and XLKO mice were injected i.p. with vehicle or PMA (500 ng/g body weight) $(68,69)$ at P9 and sacrificed 24 hours later. Blood and femurs were collected to measure serum FGF23 and phosphate levels, as well as bone FGF23 mRNA levels. PKC inhibitor Ro-32-0432 (6 mg/kg body weight) or vehicle was i.p. injected into P9 WT and Hyp mice for 24 hours. Blood was collected to measure serum FGF23 and phosphate levels.

Statistics. All data are presented as mean \pm SEM as indicated in the figure legends. The unpaired, 2 -tailed Student's $t$ test was used to determine the significance of differences between 2 groups, and 1-way ANOVA followed by Tukey's multiple-comparisons test was used to determine statistical significance among 3 or more groups. Welch's $t$ test ( 2 tailed) was used for multiple comparisons involving groups with unequal variances, followed by Bonferroni's correction (that is, $P$ values were multiplied by the number of comparisons). $P$ values smaller than $0.05\left({ }^{*} P<0.05\right.$, ${ }^{*} P<0.01$, and $\left.{ }^{* * *} P<0.001\right)$ were considered significant.

Study approval. All the animal experiments were conducted in accordance with the accepted standards of the Institutional Animal Care and Use Committee, and the studies were approved by the Massachusetts General Hospital (MGH) Subcommittee on Research Animal Care.

\section{Author contributions}

$\mathrm{QH}$ and MB designed the research. QH, LTS, JM, and CA performed the experiments. MNW, JMS, $\mathrm{RG}, \mathrm{MM}, \mathrm{AP}$, and PDP provided important reagents and tools. $\mathrm{QH}$ and $\mathrm{MB}$ interpreted the original data with feedback from all the other authors. $\mathrm{QH}$ and $\mathrm{MB}$ wrote the manuscript. 


\section{Acknowledgments}

We thank Gavin Kelsey (Babraham Institute, Cambridge, United Kingdom) and Catherine Van Raamsdonk (University of British Columbia, Vancouver, British Columbia, Canada) for providing the XLKO mice and Rosa26-floxed stop-GNAQ ${ }^{\mathrm{Q} 209 \mathrm{~L}}$ mice, respectively. We also thank HM Kronenberg (MGH and Harvard Medical School, Boston, Massachusetts, USA) for critically reviewing the manuscript. This work was supported in part by grants from the NIH/National Institute of Diabetes and Digestive and Kidney Diseases (NIDDK) (R01DK073911 to MB), NIH/National Institute of Arthritis and Musculoskeletal and Skin Diseases (NIAMS) (5K08AR067285 and R01DK116716 to MNW and R01AR05965 to PDP), and NIH/ National Institute of Dental and Craniofacial Research (R01DE13686 to MM). QH is supported by NIH/ NIDDK grant T32DK007028. JM was supported by a Boehringer Ingelheim Fonds MD fellowship. The MGH Endocrine Unit Center for Skeletal Research was funded by grant P30AR066261 from NIH/NIAMS.

Address correspondence to: Murat Bastepe, 50 Blossom St. Thier 10, Boston, Massachusetts 02114, USA. Phone: 617.726.3966; Email: bastepe@helix.mgh.harvard.edu.

1. Bergwitz C, Jüppner H. Regulation of phosphate homeostasis by PTH, vitamin D, and FGF23. Annu Rev Med. 2010;61:91-104.

2. Farrow EG, White KE. Recent advances in renal phosphate handling. Nat Rev Nephrol. 2010;6(4):207-217.

3. Quarles LD. Skeletal secretion of FGF-23 regulates phosphate and vitamin D metabolism. Nat Rev Endocrinol. 2012;8(5):276-286.

4. Carpenter TO. Primary disorders of phosphate metabolism. In: Feingold KR, et al., eds. Endotext. South Dartmouth, MA:

MDtext.com, Inc; 2018. https://www.ncbi.nlm.nih.gov/books/NBK279172/.

5. Erben RG. Physiological actions of fibroblast growth factor-23. Front Endocrinol (Lausanne). 2018;9:267.

6. ADHR Consortium. Autosomal dominant hypophosphataemic rickets is associated with mutations in FGF23. Nat Genet. 2000;26(3):345-348.

7. Shimada T, et al. Cloning and characterization of FGF23 as a causative factor of tumor-induced osteomalacia. Proc Natl Acad Sci USA. 2001;98(11):6500-6505.

8. McGrath ER, et al. Circulating fibroblast growth factor 23 levels and incident dementia: The Framingham heart study. PLoS ONE. 2019;14(3):e0213321.

9. Bonewald LF, Wacker MJ. FGF23 production by osteocytes. Pediatr Nephrol. 2013;28(4):563-568.

10. Clinkenbeard EL, et al. Conditional deletion of murine Fgf23: interruption of the normal skeletal responses to phosphate challenge and rescue of genetic hypophosphatemia. J Bone Miner Res. 2016;31(6):1247-1257.

11. Bacchetta J, Bardet C, Prié D. Physiology of FGF23 and overview of genetic diseases associated with renal phosphate wasting Metab Clin Exp. 2019;:S0026-0495(19):30021-6.

12. Wu AL, et al. Antibody-mediated activation of FGFR1 induces FGF23 production and hypophosphatemia. PLoS ONE 2013;8(2):e57322.

13. Xiao Z, Huang J, Cao L, Liang Y, Han X, Quarles LD. Osteocyte-specific deletion of Fgfr1 suppresses FGF23. PLoS ONE. 2014;9(8):e104154.

14. Han X, Xiao Z, Quarles LD. Membrane and integrative nuclear fibroblastic growth factor receptor (FGFR) regulation of FGF23. J Biol Chem. 2015;290(16):10447-10459.

15. Lavi-Moshayoff V, Wasserman G, Meir T, Silver J, Naveh-Many T. PTH increases FGF23 gene expression and mediates the high-FGF23 levels of experimental kidney failure: a bone parathyroid feedback loop. Am J Physiol Renal Physiol. 2010;299(4):F882-F889.

16. Fan Y, et al. Parathyroid hormone 1 receptor is essential to induce FGF23 production and maintain systemic mineral ion homeostasis. FASEB J. 2016;30(1):428-440.

17. Rhee Y, et al. Parathyroid hormone receptor signaling in osteocytes increases the expression of fibroblast growth factor-23 in vitro and in vivo. Bone. 2011;49(4):636-643.

18. Meir T, et al. Parathyroid hormone activates the orphan nuclear receptor Nurr1 to induce FGF23 transcription. Kidney Int 2014;86(6):1106-1115.

19. Kehlenbach RH, Matthey J, Huttner WB. XL alpha s is a new type of G protein. Nature. 1994;372(6508):804-809.

20. Hayward BE, et al. The human GNAS1 gene is imprinted and encodes distinct paternally and biallelically expressed G proteins. Proc Natl Acad Sci USA. 1998;95(17):10038-10043.

21. Peters J, et al. A cluster of oppositely imprinted transcripts at the Gnas locus in the distal imprinting region of mouse chromosome 2. Proc Natl Acad Sci USA. 1999;96(7):3830-3835.

22. Bastepe M, Gunes Y, Perez-Villamil B, Hunzelman J, Weinstein LS, Jüppner H. Receptor-mediated adenylyl cyclase activation through XLalpha(s), the extra-large variant of the stimulatory G protein alpha-subunit. Mol Endocrinol. 2002;16(8):1912-1919.

23. Linglart A, et al. Coding GNAS mutations leading to hormone resistance impair in vitro agonist- and cholera toxin-induced adenosine cyclic 3',5'-monophosphate formation mediated by human XLalphas. Endocrinology. 2006;147(5):2253-2262.

24. Liu Z, et al. Transgenic overexpression of the extra-large Gs $\alpha$ variant XL $\alpha$ s enhances Gs $\alpha$-mediated responses in the mouse renal proximal tubule in vivo. Endocrinology. 2011;152(4):1222-1233.

25. He Q, Zhu Y, Corbin BA, Plagge A, Bastepe M. The G protein $\alpha$ subunit variant XLas promotes inositol 1,4,5-trisphosphate signaling and mediates the renal actions of parathyroid hormone in vivo. Sci Signal. 2015;8(391):ra84.

26. Pignolo RJ, et al. Heterozygous inactivation of Gnas in adipose-derived mesenchymal progenitor cells enhances osteoblast differentiation and promotes heterotopic ossification. J Bone Miner Res. 2011;26(11):2647-2655.

27. Mariot V, et al. Potent constitutive cyclic AMP-generating activity of XLas implicates this imprinted GNAS product in the 
pathogenesis of McCune-Albright syndrome and fibrous dysplasia of bone. Bone. 2011;48(2):312-320.

28. Michienzi S, et al. GNAS transcripts in skeletal progenitors: evidence for random asymmetric allelic expression of Gs alpha. Hum Mol Genet. 2007;16(16):1921-1930.

29. Lau K, Willig RP, Hiort O, Hoeger PH. Linear skin atrophy preceding calcinosis cutis in pseudo-pseudohypoparathyroidism. Clin Exp Dermatol. 2012;37(6):646-648.

30. Lebrun M, et al. Progressive osseous heteroplasia: a model for the imprinting effects of GNAS inactivating mutations in humans. J Clin Endocrinol Metab. 2010;95(6):3028-3038.

31. Schuster V, Eschenhagen T, Kruse K, Gierschik P, Kreth HW. Endocrine and molecular biological studies in a German family with Albright hereditary osteodystrophy. Eur J Pediatr. 1993;152(3):185-189.

32. Schuster V, Kress W, Kruse K. Paternal and maternal transmission of pseudohypoparathyroidism type Ia in a family with Albright hereditary osteodystrophy: no evidence of genomic imprinting. J Med Genet. 1994;31(1):84.

33. Aldred MA, et al. Constitutional deletion of chromosome $20 \mathrm{q}$ in two patients affected with albright hereditary osteodystrophy. Am J Med Genet. 2002;113(2):167-172.

34. Ward S, Sugo E, Verge CF, Wargon O. Three cases of osteoma cutis occurring in infancy. A brief overview of osteoma cutis and its association with pseudo-pseudohypoparathyroidism. Australas J Dermatol. 2011;52(2):127-131.

35. Turan S, et al. Evidence of hormone resistance in a pseudo-pseudohypoparathyroidism patient with a novel paternal mutation in GNAS. Bone. 2015;71:53-57.

36. Fernández-Rebollo E, et al. Loss of XL $\alpha$ s (extra-large $\alpha$ s) imprinting results in early postnatal hypoglycemia and lethality in a mouse model of pseudohypoparathyroidism Ib. Proc Natl Acad Sci USA. 2012;109(17):6638-6643.

37. Spatz JM, et al. The Wnt inhibitor sclerostin is up-regulated by mechanical unloading in osteocytes in vitro. J Biol Chem. 2015;290(27):16744-16758.

38. He Q, et al. Large G protein $\alpha$-subunit XL $\alpha$ s limits clathrin-mediated endocytosis and regulates tissue iron levels in vivo. Proc Natl Acad Sci USA. 2017;114(45):E9559-E9568.

39. Wright MF, et al. Mechanisms of intracellular calcium homeostasis in developing and mature bovine corpora lutea. Biol Reprod. 2014;90(3):55.

40. Chen X, et al. Combined PKC and MEK inhibition in uveal melanoma with GNAQ and GNA11 mutations. Oncogene. 2014;33(39):4724-4734.

41. Chen X, et al. RasGRP3 mediates MAPK pathway activation in GNAQ mutant uveal melanoma. Cancer Cell. 2017;31(5):685-696.e6.

42. Huang JL, Urtatiz O, Van Raamsdonk CD. Oncogenic G protein GNAQ induces uveal melanoma and intravasation in mice. Cancer Res. 2015;75(16):3384-3397.

43. Durlacher-Betzer K, Hassan A, Levi R, Axelrod J, Silver J, Naveh-Many T. Interleukin-6 contributes to the increase in fibroblast growth factor 23 expression in acute and chronic kidney disease. Kidney Int. 2018;94(2):315-325.

44. Konermann S, et al. Genome-scale transcriptional activation by an engineered CRISPR-Cas 9 complex. Nature. 2015;517(7536):583-588.

45. Holm IA, Huang X, Kunkel LM. Mutational analysis of the PEX gene in patients with X-linked hypophosphatemic rickets. Am J Hum Genet. 1997;60(4):790-797.

46. Swarthout JT, Doggett TA, Lemker JL, Partridge NC. Stimulation of extracellular signal-regulated kinases and proliferation in rat osteoblastic cells by parathyroid hormone is protein kinase C-dependent. J Biol Chem. 2001;276(10):7586-7592.

47. Klemke M, Pasolli HA, Kehlenbach RH, Offermanns S, Schultz G, Huttner WB. Characterization of the extra-large G protein alpha-subunit XLalphas. II. Signal transduction properties. J Biol Chem. 2000;275(43):33633-33640.

48. Liu Z, Turan S, Wehbi VL, Vilardaga JP, Bastepe M. Extra-long Gas variant XL $\alpha$ s protein escapes activation-induced subcellular redistribution and is able to provide sustained signaling. J Biol Chem. 2011;286(44):38558-38569.

49. Forster IC, Hernando N, Biber J, Murer H. Proximal tubular handling of phosphate: A molecular perspective. Kidney Int. 2006;70(9):1548-1559.

50. Liu S, et al. Novel regulators of Fgf23 expression and mineralization in Hyp bone. Mol Endocrinol. 2009;23(9):1505-1518.

51. Zaman G, et al. Loading-related regulation of transcription factor EGR2/Krox-20 in bone cells is ERK1/2 protein-mediated and prostaglandin, Wnt signaling pathway-, and insulin-like growth factor-I axis-dependent. J Biol Chem. 2012;287(6):3946-3962.

52. Kucich U, Rosenbloom JC, Abrams WR, Bashir MM, Rosenbloom J. Stabilization of elastin mRNA by TGF-beta: initial characterization of signaling pathway. Am J Respir Cell Mol Biol. 1997;17(1):10-16.

53. Sriraman V, Modi SR, Bodenburg Y, Denner LA, Urban RJ. Identification of ERK and JNK as signaling mediators on protein kinase C activation in cultured granulosa cells. Mol Cell Endocrinol. 2008;294(1-2):52-60.

54. Caino MC, von Burstin VA, Lopez-Haber C, Kazanietz MG. Differential regulation of gene expression by protein kinase C isozymes as determined by genome-wide expression analysis. J Biol Chem. 2011;286(13):11254-11264.

55. Knab VM, et al. Acute parathyroid hormone injection increases C-terminal but not intact fibroblast growth factor 23 levels. Endocrinology. 2017;158(5):1130-1139

56. Farrow EG, et al. Iron deficiency drives an autosomal dominant hypophosphatemic rickets (ADHR) phenotype in fibroblast growth factor-23 (Fgf23) knock-in mice. Proc Natl Acad Sci USA. 2011;108(46):E1146-E1155.

57. Hori M, Kinoshita Y, Taguchi M, Fukumoto S. Phosphate enhances Fgf23 expression through reactive oxygen species in UMR106 cells. J Bone Miner Metab. 2016;34(2):132-139.

58. Takashi Y, et al. Activation of unliganded FGF receptor by extracellular phosphate potentiates proteolytic protection of FGF23 by its O-glycosylation. Proc Natl Acad Sci U S A. 2019;116(23):11418-11427.

59. Xie $\mathrm{T}$, et al. The alternative stimulatory $\mathrm{G}$ protein alpha-subunit XLalphas is a critical regulator of energy and glucose metabolism and sympathetic nerve activity in adult mice. J Biol Chem. 2006;281(28):18989-18999.

60. Plagge A, et al. The imprinted signaling protein XL alpha s is required for postnatal adaptation to feeding. Nat Genet. 2004;36(8):818-826

61. Goetz R, et al. Molecular insights into the klotho-dependent, endocrine mode of action of fibroblast growth factor 19 subfamily members. Mol Cell Biol. 2007;27(9):3417-3428.

62. Wein MN, et al. HDAC5 controls MEF2C-driven sclerostin expression in osteocytes. J Bone Miner Res. 2015;30(3):400-411. 
63. He Q, et al. Deficiency of Sef is associated with increased postnatal cortical bone mass by regulating Runx2 activity. $J$ Bone Miner Res. 2014;29(5):1217-1231.

64. Masuda M, et al. Regulation of renal sodium-dependent phosphate co-transporter genes (Npt2a and Npt2c) by all-trans-retinoic acid and its receptors. Biochem J. 2010;429(3):583-592.

65. Biber J, Stieger B, Stange G, Murer H. Isolation of renal proximal tubular brush-border membranes. Nat Protoc. 2007;2(6):1356-1359.

66. Ben-Dov IZ, et al. The parathyroid is a target organ for FGF23 in rats. J Clin Invest. 2007;117(12):4003-4008.

67. Gattineni J, et al. FGF23 decreases renal NaPi-2a and NaPi-2c expression and induces hypophosphatemia in vivo predominantly via FGF receptor 1. Am J Physiol Renal Physiol. 2009;297(2):F282-F291.

68. Dzietko M, Hahnemann M, Polley O, Sifringer M, Felderhoff-Mueser U, Bührer C. Effects of PMA (phorbol-12-myristate-13-acetate) on the developing rodent brain. Biomed Res Int. 2015;2015:318306.

69. Schneider A, Zhi X, Bartke A, Kopchick JJ, Masternak MM. Effect of growth hormone receptor gene disruption and PMA treatment on the expression of genes involved in primordial follicle activation in mice ovaries. Age (Dordr). 2014;36(4):9701. 